Pacific Journal of Mathematic 


\title{
ON SOME TRIGONOMETRIC TRANSFORMS
}

\author{
Oтto SzÁSz
}

1. Introduction. To a given series $\sum_{n=1}^{\infty} u_{n}$ we consider the transform

$$
A_{n}=\sum_{\nu=1}^{n} u_{\nu} \frac{\sin \nu t_{n}}{\nu t_{n}}, \quad \text { where } t_{n} \downarrow 0 \text { as } n \rightarrow \infty
$$

It was shown in a previous paper [ 5 , Section 4, Theorem 3] that the transform (1.1) is regular if and only if

$$
n t_{n}=O(1) \text {, }
$$

as $n \longrightarrow \infty$.

We shall now consider the transform (1.1) in relation to Cesàro means. In a forthcoming paper Cornelius Lanczos has found independently that the transform (1.1) is very useful in summing Fourier series and derived series, and gave some very interesting examples; he takes $t_{n}=\pi / n$. Of our results we quote here the following theorem:

THEOREM 1. In order that the transform (1.1) includes $(C, 1)$ summability, it is necessary and sufficient that

$$
n t_{n}=p \pi+\alpha_{n}, \quad n \alpha_{n}=O(1),
$$

p a positive integer.

We also discuss other triangular transforms which may be generated by "truncation" of well-known summation processes, such as Riemann summability. The transform $A_{n}$ and the transform $D_{n}$ (Section 5) are special cases of the general transform

$$
\gamma_{n}=\sum_{\nu=0}^{n} u_{\nu} \phi\left(\nu P_{n}\right)
$$

Received March 8, 1950. Presented to the American Mathematical Society December 30, 1948. The preparation of this paper was sponsored (in part) by the Office of Naval Research.

Pacific J. Math. 1 (1951), 291-304. 
where $\phi(P)$ is a function of the $n$-dimensional point $P\left(x_{1}, x_{2}, \cdots, x_{n}\right)$, and $P_{n} \rightarrow 0$. This transform and many special cases of it were discussed by W. Rogosinski [4]; in particular, the special case $\alpha_{n}=0$ of our Theorem 4 is included in his result on page 96 . The general approach is essentially the same as in the present paper.

2. Proof of Theorem 1. If we write

$$
\begin{aligned}
\sum_{\nu=1}^{n} u_{\nu}=s_{n}, \quad & \sum_{\nu=1}^{n} s_{\nu}=s_{n}^{\prime}, \quad \frac{\sin \nu t_{n}}{\nu t_{n}}-\frac{\sin (\nu+1) t_{n}}{(\nu+1) t_{n}}=\Delta_{\nu}, \\
& \frac{\sin \nu t_{n}}{\nu t_{n}}-\frac{2 \sin (\nu+1) t_{n}}{(\nu+1) t_{n}}+\frac{\sin (\nu+2) t_{n}}{(\nu+2) t_{n}}=\Delta_{\nu}^{2},
\end{aligned}
$$

then

$$
\begin{aligned}
A_{n} & =\sum_{\nu=1}^{n-1} s_{\nu} \Delta_{\nu}+s_{n} \frac{\sin n t_{n}}{n t_{n}} \\
& =\sum_{\nu=1}^{n-2} s_{\nu}^{\prime} \Delta_{\nu}^{2}+s_{n-1}^{\prime} \Delta_{n-1}+\left(s_{n}^{\prime}-s_{n-1}^{\prime}\right) \frac{\sin n t_{n}}{n t_{n}},
\end{aligned}
$$

or

$$
\begin{aligned}
& A_{n}=\sum_{\nu=1}^{n-2} s_{\nu}^{\prime} \Delta_{\nu}^{2}+s_{n-1}^{\prime}\left[\frac{\sin (n-1) t_{n}}{(n-1) t_{n}}-\frac{2 \sin n t_{n}}{n t_{n}}\right] \\
& +s_{n}^{\prime} \frac{\sin n t_{n}}{n t_{n}} \text {. }
\end{aligned}
$$

Now (C. 1) summability of $\sum_{n=1}^{\infty} u_{n}$ to $s$ means that

$$
n^{-1} s_{n}^{\prime} \rightarrow s,
$$
as $n \longrightarrow \infty$.

If $s_{n} \equiv 1$, then $A_{n}=\sin t_{n} / t_{n} \rightarrow 1$.

In order that (2.2) imply $A_{n} \rightarrow s$, it is necessary and sufficient [in view of (2.1)] that 


$$
\frac{\sin n t_{n}}{t_{n}}=O(1), \quad \frac{\sin (n-1) t_{n}}{t_{n}}=O(1)
$$

$$
\sum_{\nu=1}^{n-2} \nu\left|\Delta_{\nu}^{2}\right|=O(1),
$$

as $n \rightarrow \infty$.

The first condition of (2.3) [in view of (1.2)] is equivalent to

$$
\sin n t_{n}=O\left(t_{n}\right)=O(1 / n)
$$

hence

$$
n t_{n}=p \pi+\alpha_{n}, \quad n \alpha_{n}=O(1) .
$$

The second condition of (2.3) now reduces to

$$
\cos n t_{n} \sin t_{n}=O\left(t_{n}\right)
$$

or

$$
\cos \alpha_{n} \sin t_{n}=O\left(n^{-1}\right),
$$

which is satisfied. Finally

$$
\frac{\sin \nu t}{\nu}=\int_{0}^{t} \cos \nu x d x=R \int_{0}^{t} e^{i \nu x} d x
$$

hence

$$
t_{n} \Delta_{\nu}^{2}=R \quad \int_{0}^{t_{n}} \Delta^{2} e^{i \nu x} d x=R \int_{0}^{t_{n}} e^{i \nu x}\left(1-e^{i x}\right)^{2} d x
$$

and

$$
\begin{aligned}
t_{n}\left|\Delta_{\nu}^{2}\right|<\int_{0}^{t_{n}}\left|1-e^{i x}\right|^{2} d x= & 4 \int_{0}^{t_{n}}(\sin x / 2)^{2} d x \\
& <\int_{0}^{t_{n}} x^{2} d x<t_{n}^{3} .
\end{aligned}
$$

It follows that

$$
\sum_{\nu=1}^{n-2} \nu\left|\Delta_{\nu}^{2}\right|<t_{n}^{2} \sum_{\nu=1}^{n} \nu<n^{2} t_{n}^{2}=O(1), \quad \text { as } n \rightarrow \infty .
$$


This proves Theorem 1 .

We can show by an example that the transform $A_{n}$ may be more powerful than $(C, 1)$. In (1.3) let $p=1, n a_{n}=-\pi / 2$; the series $\sum_{\nu=1}^{\infty}(-1)^{n-1} n$ (that is, $\left.u_{n}=(-1)^{n} n\right)$ is not summable $(C, 1)$, but summable $(C, 2)$ to $1 / 4$. Now

$$
\begin{aligned}
t_{n} A_{n}=\sum_{\nu=1}^{n}(-1)^{\nu-1} \sin \nu t_{n} \\
\quad=\frac{\sin t_{n}-(-1)^{n}\left[\sin n t_{n}+\sin (n+1) t_{n}\right]}{\left|1+e^{i t}\right|^{2}},
\end{aligned}
$$

where $n t_{n}=\pi-\pi / 2 n$. Hence, as $n \rightarrow \infty$,

$$
A_{n} \sim 1 / 4+o(1)
$$

An even more striking example is $u_{n}=(-1)^{n-1} n^{2}$.

3. Summation by harmonic polynomials. We get a more powerful method if we introduce the harmonic polynomial

$$
h_{n}(\rho, t)=\sum_{\nu=1}^{n} u_{\nu} \rho^{\nu} \frac{\sin \nu t}{\nu},
$$

and the corresponding transform

$$
B_{n}=\sum_{\nu=1}^{n} u_{\nu} \rho_{n}^{\nu} \frac{\sin \nu t_{n}}{\nu t_{n}}, \quad \rho_{n} \longrightarrow 1, \quad t_{n} \downarrow 0
$$

or

$$
B_{n}=t_{n}^{-1} h_{n}\left(\rho_{n}, t_{n}\right) \text {. }
$$

Let

$$
s_{n}^{k}=\sum_{\nu=0}^{n} s_{\nu} \gamma_{n-\nu}^{k-1}
$$

where 


$$
y_{n}^{k}=\frac{(k+1) \cdots(k+n)}{n !} \sim \frac{n^{k}}{\Gamma(k+1)}
$$

we also write

$$
\Delta^{k} v_{\nu}=\sum_{r=1}^{k}(-1)^{r}\left(\begin{array}{l}
k \\
r
\end{array}\right) v_{\nu+r}
$$

and

$$
\sigma_{n}^{k}=\frac{s_{n}^{k}}{\gamma_{n}^{k}}
$$

Now $(C, k)$ summability of the sequence $\left\{s_{n}\right\}$ to $s$ is defined by

$$
\lim _{n \rightarrow \infty} \sigma_{n}^{k}=s \text {. }
$$

We quote the following elementary theorem [cf. 6, Theorem 1], which is included in a more general result of Mazur [1, Theorem X] :

LEMMA 1. Let $k$ be a given positive integer, and let

$$
T_{n}=\sum_{\nu=n}^{n} a_{n, \nu} s_{\nu}, \quad n=0,1,2, \cdots
$$

In order that $\lim T_{n}$ exist, whenever the sequence $\left\{s_{n}\right\}$ is $(C, k)$ summable to $s$, it is necessary and sufficient that:

$$
\begin{array}{lr}
\sum_{\nu=0}^{n} \gamma_{\nu}^{k}\left|\Delta^{k} a_{n, \nu}\right|=O(1), & a_{n, \nu}=0 \text { for } \nu>n ; \\
\lim _{n \rightarrow \infty} \gamma_{\nu}^{k} \Delta a_{n, \nu}=\alpha_{\nu} \text { exists }, & \nu=0,1,2, \cdots ;
\end{array}
$$

$$
\lim _{n \rightarrow \infty} \sum_{\nu=0}^{n} a_{n, \nu}=\beta \text { exists. }
$$

We then have $\lim T_{n}=\beta_{s}+\sum_{\nu=0}^{\infty} \alpha_{\nu}\left(\sigma_{\nu}^{k}-s\right)$. Since then the transform $T_{n}$ 
is convergence preserving we must have (3.5) and:

$$
\lim _{n \rightarrow \infty} a_{n, \nu} \text { exists, } \quad \nu=0,1,2, \cdots \text {; }
$$

hence (3.4) and (3.5) hold, so that the conditions of Lemma 1 reduce to (3.3). In the case of the transform $B_{n}$, we have

$$
\begin{aligned}
& a_{n, n}=\rho_{n}^{n} \frac{\sin n t_{n}}{n t_{n}}, \\
& a_{n, \nu}=\rho_{n}^{\nu} \frac{\sin \nu t_{n}}{\nu t_{n}}-\rho_{n}^{\nu+1} \frac{\sin (\nu+1) t_{n}}{(\nu+1) t_{n}}, \quad \nu=1,2, \cdots \cdot n-1 ;
\end{aligned}
$$

hence

$$
a_{n, \nu} \longrightarrow 0,
$$

To satisfy (3.3) we must have

and

$$
\begin{gathered}
n^{k} \rho_{n}^{n} \frac{\sin n t_{n}}{n t_{n}}=O(1), \\
n^{k} \rho_{n}^{n-1} \frac{\sin (n-1) t_{n}}{(n-1) t_{n}}=O(1), \\
\cdot \cdot \cdot \\
n^{k} \rho_{n}^{n-k} \frac{\sin (n-k) t_{n}}{(n-k) t_{n}}=O(1),
\end{gathered}
$$

$$
\sum_{\nu=1}^{n-k-1} \nu^{k}\left|\Delta^{k+1} \rho_{n}^{\nu} \frac{\sin \nu t_{n}}{\nu t_{n}}\right|=O(1)
$$

Assume first that $k=0$; then our conditions become :

$$
\rho_{n}^{n} \frac{\sin n t_{n}}{n t_{n}}=O(1),
$$

and

$$
\sum_{\nu=1}^{n-1} \rho_{n}^{\nu}\left|\frac{\sin \nu t_{n}}{\nu t_{n}}-\rho_{n} \frac{\sin (\nu+1) t_{n}}{(\nu+1) t_{n}}\right|=O(1) .
$$


We now prove the lemma:

LEMMA 2. If

$$
\rho_{n}^{n}=O(1), \quad \frac{1-\rho_{n}^{n}}{1-\rho_{n}} t_{n}=O(1), \quad \text { as } t_{n} \downarrow 0, \quad \rho_{n} \rightarrow 1
$$

then $B_{n}$ is a regular transform.

Clearly (3.9) holds, and we need only to show that (3.10) also holds.

If $\rho_{n}>1$, then $\rho_{n}^{\nu}<\rho_{n}^{n}, \nu=0,1, \cdots, n-1$; if on the other hand $\rho_{n} \leq 1$, then $\rho_{n}^{\nu} \leq 1$. Hence, in either case,

$$
\max _{0 \leq \nu \leq n} \rho_{n}^{\nu}=O(1), \quad \text { as } n \rightarrow \infty \text {. }
$$

We have

$$
\begin{aligned}
\sum_{\nu=1}^{n} \rho^{\nu}\left|\frac{\sin \nu t}{\nu}-\rho \frac{\sin (\nu+1) t}{\nu+1}\right| & \leq \sum_{\nu=1}^{n} \rho^{\nu}\left|\frac{\sin \nu t}{\nu}-\frac{\sin (\nu+1) t}{\nu+1}\right| \\
& +(1-\rho) \sum_{\nu=1}^{n}\left|\frac{\sin (\nu+1) t}{\nu+1}\right| \rho^{\nu} ;
\end{aligned}
$$

the second term is $O(t)$, and

$$
\frac{\sin \nu t}{\nu}-\frac{\sin (\nu+1) t}{\nu+1}=\int_{0}^{t}[\cos \nu x-\cos (\nu+1) x] d x=O\left(t^{2}\right)
$$

so that

$$
\sum_{\nu=1}^{n} \rho^{\nu}\left|\frac{\sin \nu t}{\nu}-\frac{\sin (\nu+1) t}{\nu+1}\right|=O\left(t^{2} \frac{1-\rho^{n}}{1-\rho}\right) .
$$

Thus (3.10) is satisfied and Lemma 2 holds.

Note that the condition $\rho_{n}^{n}=O(1)$ is equivalent to $n\left(\rho_{n}-1\right)<c$, a positive constant (see $[5, \mathrm{p} .73])$; furthermore, if $n t_{n}=O(1)$, then clearly the second condition of (3.11) holds.

Next let $k=1$; we shall prove the theorem: 
THEOREM 2. If (3.11) holds, and if

$$
\rho_{n}^{n} \sin n t_{n}=O\left(t_{n}\right),
$$$$
n \rightarrow \infty,
$$

then $B_{n}$ includes $(C, 1)$.

The conditions (3.6)-(3.8) now become:

$$
\begin{aligned}
\rho_{n}^{n} \sin n t_{n} & =O\left(t_{n}\right), \\
\rho_{n}^{n} \sin (n-1) t_{n} & =O\left(t_{n}\right),
\end{aligned}
$$

and

$$
\sum_{\nu=1}^{n-2} \nu\left|\triangle^{2} \rho_{n}^{\nu} \frac{\sin \nu t_{n}}{\nu}\right|=O\left(t_{n}\right)
$$

Clearly, we need only to show that (3.13) is satisfied. Now

$$
\begin{aligned}
\Delta^{2} \rho^{\nu} \frac{\sin \nu t}{\nu}=\Delta^{2} \rho^{\nu} \int_{0}^{t} \cos \nu x d x & =R \Delta^{2} \int_{0}^{t} \rho^{\nu} e^{i \nu x} d x \\
=R \int_{0}^{t} \rho^{\nu} e^{i \nu x}\left(1-2 \rho e^{i x}+\rho^{2} e^{2 i x}\right) d x & \\
& =R \int_{0}^{t} \rho^{\nu} e^{i \nu x}\left(1-\rho e^{i x}\right)^{2} d x .
\end{aligned}
$$

Hence

$$
\left|\Delta^{2} \rho^{\nu} \frac{\sin \nu t}{\nu}\right|<\rho^{\nu} \int_{0}^{t}\left|1-\rho e^{i x}\right|^{2} d x<\rho^{\nu} t\left\{(1-\rho)^{2}+\rho t^{2}\right\} ;
$$

it follows from (3.11) that

$$
\sum_{\nu=1}^{n} \nu\left|\Delta^{2} \rho_{n}^{\nu} \frac{\sin \nu t_{n}}{\nu t_{n}}\right|<\left\{\left(1-\rho_{n}\right)^{2}+\rho_{n} t_{n}^{2}\right\} \sum_{\nu=1}^{n} \nu \rho_{n}^{\nu}=O(1) .
$$

This proves (3.13) and Theorem 2.

4. Comparison of $B_{n}$ and $(C, k), k \geq 2$. We wish to prove the following theorem : 
THE OREM 3. Suppose that (3.11) holds and that

$$
\begin{aligned}
& n^{k-1} \rho_{n}^{n} \sin n t_{n}=O\left(t_{n}\right), \\
& n^{k-1} \rho_{n}^{n} \cos n t_{n}=O(1), \quad \rho_{n} \rightarrow 1, \quad t_{n} \downarrow 0,
\end{aligned}
$$

then $B_{n}$ includes $(C, k)$ summability.

Now (3.6) holds because of (4.1), and then (3.7) follows from (4.2). It remains to prove (3.8). We have

$$
\begin{array}{rl}
\Delta^{k+1} \rho^{\nu} \frac{\sin \nu t}{\nu}=\Delta^{k+1} \rho^{\nu} \int_{0}^{t} \cos \nu x & d x=\Delta^{k+1} R \int_{0}^{t} \rho^{\nu} e^{i \nu x} d x \\
= & R \int_{0}^{t} \rho^{\nu} e^{i \nu x}\left(1-\rho e^{i x}\right)^{k+1} d x ;
\end{array}
$$

hence

$$
\begin{aligned}
&\left|\Delta^{k+1} \rho^{\nu} \frac{\sin \nu t}{\nu}\right|<\rho^{\nu} \int_{0}^{t}\left|1-\rho e^{i x}\right|^{k+1} d x \\
&<\rho^{\nu} \int_{0}^{t}\left\{(1-\rho)^{2}+\rho t^{2}\right\}^{(k+1) / 2} d x \\
&=O\left(\rho^{\nu} t\left\{(1-\rho)^{k+1}+t^{k+1}\right\}\right) .
\end{aligned}
$$

It follows that

$$
\begin{aligned}
\sum_{\nu=1}^{n} \nu^{k}\left|\Delta^{k+1} \rho_{n}^{\nu} \frac{\sin \nu t_{n}}{\nu t_{n}}\right|=O\left(\sum_{\nu=1}^{n} \nu^{k} \rho_{n}^{\nu}\left\{\left(1-\rho_{n}\right)^{k+1}+t_{n}^{k+1}\right\}\right) \\
=O\left(\left(1-\rho_{n}\right)^{k+1} \sum_{\nu=1}^{n} \nu^{k} \rho_{n}^{\nu}\right)+O\left(t_{n}^{k+1} \sum_{\nu=1}^{n} \nu^{k} \rho_{n}^{\nu}\right) .
\end{aligned}
$$

Here the first term is $O(1)$ by Lemma 2 of [6]; finally

$$
t_{n}^{k+1} \sum_{\nu=1}^{n} \nu^{k} \rho_{n}^{\nu}=O\left(t_{n} \sum_{\nu=1}^{n} \rho_{n}^{\nu}\right)^{k+1}=O(1)
$$


This proves Theorem 3 .

An interesting special case is $t_{n}=\pi / n$; the conditions now reduce to the single condition

$$
n^{k-1} \rho_{n}^{n}=O(1)
$$

If, in particular, $n^{k} \rho_{n}^{n}=O(1)$ for all $k$, then $B_{n}$ includes all $(C, k)$.

Observe that by Lemma 1 of [6] the condition $n^{k} \rho_{n}^{n}=O(1)$ is equivalent to

$$
\lim \sup \left\{n\left(\rho_{n}-1\right)+k \log n\right\}<+\infty .
$$

Note also that (4.1) and (4.2) imply:

$$
n^{k-1} \rho_{n}^{n}=O(1)
$$

5. Truncated Riemann summability. The series $\sum_{\nu=0}^{\infty} u_{\nu}$ is called $(R, k)$ summable to $s$ if the series

$$
u_{0}+\sum_{n=1}^{\infty}\left(\frac{\sin n t}{n t}\right)^{k} u_{n}=R_{k}(t)
$$

converges in some interval $0<t<t_{0}$, and if

$$
R_{k}(t) \rightarrow s \text {, }
$$

as $t \rightarrow 0$.

For $k=1$ it is sometimes called Lebesgue summability. The method $(R, k)$ is regular for $k \geq 2$ and, in fact, it is more powerful than $(C, k-2)$; for $k=2$, it was employed by Riemann in the theory of trigonometric series. We generate from it by truncation the triangular series to sequence transform $\left(u_{0}=0\right)$ :

$$
D_{n}=\sum_{\nu=1}^{n} u_{\nu}\left(\frac{\sin \nu t_{n}}{\nu t_{n}}\right)^{k}=\sum_{\nu=1}^{n-1} s_{\nu} \Delta\left(\frac{\sin \nu t_{n}}{\nu t_{n}}\right)^{k}+s_{n}\left(\frac{\sin n t_{n}}{n t_{n}}\right)^{k} ;
$$

$k$ is a positive integer. We assume $k \geq 2$; it is then easy to show that $D_{n}$ is a regular transformation.

From Lemma 1 we find for $(C, k)$ to be included in $D_{n}$ the conditions :

$$
\begin{array}{ll}
t_{n}^{-k}\left(\sin \overline{n-\nu} t_{n}\right)^{k}=O(1), \quad & \text { for } \nu=0,1, \cdots, k ; \\
\sum_{\nu=1}^{n-k-1} \nu^{k}\left|\Delta^{k+1}\left(\frac{\sin \nu t_{n}}{\nu t_{n}}\right)^{k}\right|=O(1), & n \rightarrow \infty .
\end{array}
$$


It follows from (5.2) (see Section 2) that we must have

$$
n t_{n}=p \pi+\alpha_{n}, \quad n \alpha_{n}=O(1), \quad \quad p \text { a positive integer }
$$

now $(5.2)$ reduces to

$$
t_{n} \sin \left(\alpha_{n}-\nu t_{n}\right)=O(1), \quad \nu=0,1, \cdots, k,
$$

and this is satisfied in view of (5.4).

To show that now (5.3) also holds, we employ a lemma, due to Obreschkoff $[2, \mathrm{p} .443]$ :

LEMMA 3. We have

$$
\left|\Delta^{m}\left(\frac{\sin \nu t}{\nu t}\right)^{k}\right| \leq M \frac{t^{m-k}}{\nu^{k}}
$$

where $M$ is independent of $t$ and $\nu$.

It now follows that

$$
\sum_{\nu=1}^{n} \nu^{k}\left|\Delta^{k+1}\left(\frac{\sin \nu t_{n}}{\nu t_{n}}\right)^{k}\right|=O\left(n t_{n}\right)=O(1), \quad n \rightarrow \infty
$$

This yields the following theorem:

THEOREM 4. If $n t_{n}=p \pi+\alpha_{n}, p$ a positive integer, $n \alpha_{n}=O(1)$, then the trans form

$$
\sum_{\nu=1}^{n} u_{\nu}\left(\frac{\sin \nu t_{n}}{\nu t_{n}}\right)^{k}=D_{n}
$$

includes $(C, k)$ summability ( $k$ a positive integer).

6. A converse theorem. We shall establish the following result.

THEOREM 5. If

$$
\lim \inf \left|\frac{\sin n t_{n}}{n t_{n}}\right|^{k}=\lambda>1 / 2
$$

then the transform $D_{n}$ is equivalent to convergence. 
It follows from (6.1) that lim sup $n t_{n}<2^{1 / k}$; hence (see Sections 1 and 5) the transform $D_{n}$ is regular. We now wish to show that $D_{n} \rightarrow s$ implies $s_{n} \rightarrow s$; we follow a device used by R. Rado [3].

Assume first that $s=0$, and that $s_{n}=0(1)$; then

$$
0 \leq \limsup _{n \rightarrow \infty}\left|s_{n}\right|=\delta<\infty,
$$

and we shall show that $\delta=0$. To a given $\epsilon>0$ choose $n=n(\epsilon)$ so that $\left|s_{\nu}\right|<$ $\delta+\epsilon$ for $\nu \geq n$. Next choose $m>n$ and such that $\left|s_{m}\right|>\delta-\epsilon$. We have

$$
s_{m}\left(\frac{\sin m t_{m}}{m t_{m}}\right)^{k}=D_{m}-\sum_{\nu=1}^{m-1} s_{\nu} \Delta_{\nu},
$$

where

$$
\Delta_{\nu}=\left(\frac{\sin \nu t_{m}}{\nu t_{m}}\right)^{k}-\left(\frac{\sin (\nu+1) t_{m}}{(\nu+1) t_{m}}\right)^{k} ;
$$

hence, as $m t_{m}<\pi$, we have

$$
\begin{aligned}
\left.\left|s_{m}\right| \frac{\sin m t_{m}}{m t_{m}}\right|^{k} & <\left|D_{m}\right|+\left|\sum_{\nu=1}^{n-1} s_{\nu} \Delta_{\nu}\right|+\left|\sum_{\nu=n}^{m-1} s_{\nu} \Delta_{\nu}\right| \\
& <o(1)+(\delta+\epsilon)\left\{\left(\frac{\sin n t_{m}}{n t_{m}}\right)^{k}-\left(\frac{\sin m t_{m}}{m t_{m}}\right)^{k}\right\} .
\end{aligned}
$$

It follows that

$$
\delta-\epsilon<\left|s_{m}\right|<o(1)+(\delta+\epsilon)\{1 / \lambda-1+o(1)\} .
$$

But $1 / \lambda<2$, and $\epsilon$ is arbitrarily small; hence $\delta=0$.

We next assume $s=0$ and $\lim \sup \left|s_{n}\right|=\infty$; choose $\epsilon>0$ and $\omega$ large. Denote by $m=m(\omega)$ the least $m$ for which $\left|s_{m}\right|>\omega$; then

$$
\omega<\left|s_{m}\right|<o(1)+\omega\{1 / \lambda-1+o(1)\} .
$$

But this is impossible for $\lambda>1 / 2$, small $\epsilon$, and large $m$. This proves our theorem for $s=0$. Finally, applying this result to the sequence $\left\{s_{n}-s\right\}$ and its transform completes the proof of Theorem 5 .

7. Application to Fourier series. Suppose that $f(x)$ is a Lebesgue integrable 
function of period $2 \pi$, and let

$$
f(x) \sim a_{0} / 2+\sum_{n=1}^{\infty}\left(a_{n} \cos n x+b_{n} \sin n x\right) \equiv \sum u_{n}(x) ;
$$

we may assume here $a_{0}=0$. Now (cf. $[7$, p. 27])

$$
F(x)=\int_{0}^{x} f(t) d t=C+\sum_{n=1}^{\infty}\left(a_{n} \sin n x-b_{n} \cos n x\right) \frac{1}{n},
$$

where

$$
C=\sum_{n=1}^{\infty} \frac{1}{n} b_{n}
$$

It is known $[7, \mathrm{p} .55]$ that at every point $x$ where $F^{\prime}(x)$ exists and is finite, the series $(6.1)$ is summable $(C, r), r>1$, to the value $F^{\prime}(x)$.

It now follows from Theorem 3 for $k=2$ and $t_{n}=\pi / n$ that if $n \rho_{n}^{n}=O(1)$, then

$$
\sum_{\nu=1}^{n} u_{\nu}(x) \rho_{n}^{\nu} \frac{\sin \nu \pi / n}{\nu \pi / n} \rightarrow F^{\prime}(x)
$$

Furthermore, Theorem 4 yields, for $k=2$, that if

$$
n t_{n}=p \pi+\alpha_{n}, \quad n \alpha_{n}=O(1)
$$

then

$$
\sum_{\nu=1}^{n} u_{\nu}(x)\left(\frac{\sin \nu t_{n}}{\nu t_{n}}\right)^{2} \rightarrow F^{\prime}(x)
$$

An analogous theorem holds for higher derivatives (cf. [7, p. 257]). 


\section{REFERENCES}

1. St. Mazur, Über lineare Limitierungsverfahren, Math. Z. 28 (1928), 599-611.

2. N. Obreschkoff, Über das Riemannsche Summierungsverfahren, Math. Z. 48 (194243), 441-454.

3. R. Radó, Some elementary Tauberian theorems (I), Quart. J. Math., Oxford Ser. 9 (1938), 274-282.

4. W. Rogosinski, Abschnittsverhalten bei trigonometrischen und Fourierschen Reihen, Math. Z. 41 (1936), 75-136.

5. Otto Szász, Some new summability methods with applications, Ann. of Math. 43 (1942), 69-83.

6. - On some summability methods with triangular matrix, Ann. of Math. 46 (1945), 567-577.

7. A. Zygmund, Trigonometrical series, Monografje Matematyczne, Warszawa-Lwow, 1935.

National Bureau of Standards, Los Angeles 


\title{
PACIFIC JOURNAL OF MATHEMATICS
}

\section{EDITORS}

\author{
Herbert BuSEMANN \\ R. M. RoBINSON \\ University of Southern California \\ University of California \\ Los Angeles 7, California \\ Berkeley 4, California \\ E. F. BEC KENBACH, Managing Editor \\ University of California \\ Los Angeles 24, California
}

\section{ASSOCIATE EDITORS}
R. P. DILWORTH
P. R. HALMOS
BØRGE JESSEN
J. J. STOKER
HERBERT FEDERER
HEINZ HOPF
PAUL LÉVY
MARSHALL HALL
R. D. JAMES
GEORGE PÓLYA
E. G. STRAUS
KÖSAKU YOSIDA

\section{SPONSORS}

UNIVERSITY OF BRITISH COLUMBIA

CALIFORNIA INSTITUTE OF TECHNOLOGY

UNIVERSITY OF CALIFORNIA, BERKELEY

UNIVERSITY OF CALIFORNIA, DAVIS

UNIVERSITY OF CALIFORNIA, LOS ANGELES

UNIVERSITY OF CALIFORNIA, SANTA BARBARA

OREGON STATE COLLEGE

UNIVERSITY OF OREGON
UNIVERSITY OF SOUTHERN CALIFORNIA

STANFORD UNIVERSITY

WASHINGTON STATE COLLEGE

UNIVERSITY OF WASHINGTON

AMERICAN MATHEMATICAL SOCIETY

NATIONAL BUREAU OF STANDARDS, INSTITUTE FOR NUMERIGAL ANALYSIS

Mathematical papers intended for publication in the Pacific Journal of Mathematics should be typewritten (double spaced), and the author should keep a complete copy. Manuscripts may be sent to any of the editors. All other communications to the editors should be addressed to the managing editor, E. F. Beckenbach, at the address given above.

Authors are entitled to receive 100 free reprints of their published papers and may obtain additional copies at cost.

The Pacific Journal of Mathematics is published quarterly, in March, June, September, and December. The price per volume (4 numbers) is $\$ 8.00$; single issues, $\$ 2.50$. Spécial price to individual faculty members of supporting institutions and to members of the American Mathematical Society: $\$ 4.00$ per volume; single issues, $\$ 1.25$.

Subscriptions, orders for back numbers, and changes of address should be sent to the publishers, University of California Press, Berkeley 4, California.

UNIVERSITY OF CALIFORNIA PRESS - BERKELEY AND LOS ANGELES 


\section{Pacific Journal of Mathematics}

\section{Vol. 1, No. $2 \quad$ December, 1951}

Tom M. (Mike) Apostol, On the Lerch zeta function ................. 161

Ross A. Beaumont and Herbert S. Zuckerman, A characterization of the subgroups of the additive rationals ....................... 169

Richard Bellman and Theodore Edward Harris, Recurrence times for the Ehrenfest model................................... 179

Stephen P.L. Diliberto and Ernst Gabor Straus, On the approximation of a function of several variables by the sum of functions of fewer

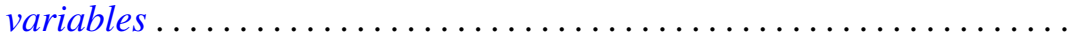

Isidore Isaac Hirschman, Jr. and D. V. Widder, Convolution transforms with complex kernels ................................ 211

Irving Kaplansky, A theorem on rings of operators .............. 227

W. Karush, An iterative method for finding characteristic vectors of a symmetric matrix............................... 233

Henry B. Mann, On the number of integers in the sum of two sets of positive integers ......................................... 249

William H. Mills, A theorem on the representation theory of Jordan

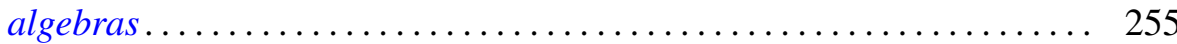

Tibor Radó, An approach to singular homology theory.............. 265

Otto Szász, On some trigonometric transforms ................... 291

James G. Wendel, On isometric isomorphism of group algebras ......... 305

George Milton Wing, On the $L^{p}$ theory of Hankel transforms ... 